\title{
Corela
}

Cognition, représentation, langage

HS-26 | 2018

Réseaux communautaires et variation dans le langage en moyen français et français préclassique

\section{La notion de variation dans le langage : quelques repères}

Pierre Larrivée

\section{CpenEdition}

Journals

Édition électronique

URL : http://journals.openedition.org/corela/6650

DOI : $10.4000 /$ corela. 6650

ISSN : 1638-573X

Éditeur

Cercle linguistique du Centre et de l'Ouest - CerLICO

Référence électronique

Pierre Larrivée, «La notion de variation dans le langage : quelques repères », Corela [En ligne], HS-26 | 2018, mis en ligne le 14 novembre 2018, consulté le 30 avril 2019. URL : http:// journals.openedition.org/corela/6650 ; DOI : 10.4000/corela.6650

Ce document a été généré automatiquement le 30 avril 2019

\section{cc) (i)}

Corela - cognition, représentation, langage est mis à disposition selon les termes de la licence Creative Commons Attribution - Pas d'Utilisation Commerciale - Partage dans les Mêmes Conditions 4.0 International. 


\title{
La notion de variation dans le langage : quelques repères
}

\author{
Pierre Larrivée
}

\section{Langue et variation}

1 La recherche sur le langage a pour objectif d'établir les généralités qui sous-tendent les manifestations d'une langue, voire du langage. Cet objectif est en phase avec la perspective de Saussure selon qui

La langue est un trésor déposé par la pratique de la parole dans les sujets appartenant à une même communauté, un système grammatical existant virtuellement dans chaque cerveau, ou plus exactement, dans les cerveaux d'un ensemble d'individus; car la langue n'est complète dans aucun, elle n'existe parfaitement que dans la masse. (De Saussure 1972 : 30)

C'est dans l'entier du réseau ou groupe qu'existent les généralités déployant le système linguistique. Cela pose la question de savoir où "dans la masse» existe cette connaissance. L'autre question primordiale est de savoir comment cette connaissance collective rend compte des pratiques différenciées des locuteurs, autrement dit des variations individuelles ou communautaires. En effet, le passage de Saussure reconnait la tension entre les pratiques de (réseaux d') individus et un ensemble de connaissances homogènes qui constituent le "trésor d'une langue ». C'est d'avoir fait l'impasse sur la première partie de l'équation qui a été sévèrement reproché à Saussure par la sociolinguistique européenne naissante, laquelle comme son homologue américaine, souligne l'hétérogénéité des pratiques langagières.

3 La variation dans le langage se manifeste en l'occurrence sous différents rapports, dont rendent compte les termes diatopique (référant à la région), diastratie (selon la dimension sociale) et diaphasie (stylistique ou situationnel) et diachronie. Cette hétérogénéité se retrouve dans la grammaire et est facilement identifiable dans le lexique. Par exemple, la forme grammaticale de l'interrogation en français contient des variables régionales (le $t i$ illustré par ça va-ti ? étant encore attesté en Normandie par exemple), sociales (l'étude quantitative de Behnstedt 1978 montrant qu'une interrogative de la forme Quand que c'est 
qu'il arrive? qu'il atteste dans la pratique des membres des classes travailleuses ne se retrouve pas dans celle des classes moyennes), et stylistiques (l'inversion du sujet encore présente dans les textes littéraires a disparu de l'usage ordinaire : Lefeuvre et RossiGensane ne relèvent aucune occurrence de avez-vous dans le CFPP2000, alors que dans Frantext j'en ai relevé 50 dans Frantext depuis 2010 face à 59 vous avez interrogatifs).

Ces observations appellent plusieurs remarques. D'une part, les domaines de variation sont imbriqués: le -ti normand est aussi un fait stylistique relevant de la pratique vernaculaire du français, qui sera évité dans les interactions normées; la perte de l'inversion du sujet en interrogative est aussi un phénomène d'évolution historique.

D'autre part, les marqueurs de variation sont récurrents, et cette récurrence se manifeste dans une communauté ou un type de situation. C'est dire que la variation n'est pas une production individuelle. Il y a bien quelques attestations anecdotiques de pratiques idiolectales. Levinson (2006) discute le cas de Kpemuwo, l'unique individu sourd de sa communauté villageoise isolée de l'île Rossel de Papouasie-Nouvelle Guinée. La langue des signes que ce sujet a développé lui permet d'être compris, y compris par Levinson avec qui il n'a aucune langue en commun. Une de mes tantes née en moyenne Mauricie au Québec autour de 1935 disait «si ça vient que » au sens de "s'il arrive que », sans que j'aie pu attester cet usage chez les membres de son entourage. Je n'ai jamais été témoin de ce que cet emploi ait été l'objet d'une incompréhension ou d'un commentaire métalinguistique. Pour rester dans le domaine familial, le seul exemple d'incompréhension dont j'ai été témoin portait sur l'emploi de gros comme quantifieur ( Il travaillait gros »), régulier et fréquent dans ce groupe familial, et qui n'ayant pas été compris par une francophone européenne, a amené la locutrice à reformuler ( $\mathrm{Il}$ travaillait beaucoup »). La variation idiolectale existe sans doute, mais comme la variation sociale, elle s'inscrit dans le cadre des contraintes récurrentes. Si l'examen de l'usage de traits grammaticaux du français par des locuteurs individuels montre des variabilités d'usage, ces variabilités se retrouvent soumises aux mêmes contraintes (Iglesias et Larrivée 2014 et les références qui y sont citées). La variation se retrouve donc à travers la langue, mais c'est une variation régulière, comme le propose Labov, reposant sur des paramètres récurrents et partagés. C'est ainsi que les variantes ne sont pas enclavées : aucune des variables n'est incompréhensibles aux membres des autres réseaux d'une même communauté linguistique, et le locuteur de classe moyenne comprend très bien ce que Quand est-ce que c'est qu'il arrive? veut dire. Les éventuelles productions individuelles qui ne s'appuieraient pas sur des contraintes générales ne seraient plus du langage, car elles ne seraient plus repérables comme fait partagé dans le réseau ou à un niveau général.

6 Les faits de pratiques linguistiques servent aussi à marquer l'appartenance à un réseau de locuteurs que réunit des dimensions identitaires ou d'intérêts. On peut citer l'exemple de Martha's Vineyard :

My first research was on the little island of Martha's Vineyard off Cape Cod. My friend Murray Lerner, the film maker, invited me up there. There I noticed a peculiar way of pronouncing the words right, ice, sight, with the vowel in the middle of the mouth, that was stronger among young people, but varied a great deal by occupation, by island locale, or by the speaker's background--Yankee, Portuguese, or Indian. (...)

As I finally figured out, the Martha's Vineyard sound change was serving as a symbolic claim to local rights and privileges, and the more someone tried to 
exercise that claim, the stronger was the change. This became my M.A. essay, and I gave it as a paper before the Linguistic Society of America. (Labov 1987)

Dans cette recherche, Labov montre que sur l'île de Martha's Vineyard, la prononciation de certaines voyelles est corrélée à un positionnement idéologique, celui de l'appartenance à la communauté insulaire, permettant de se démarquer des villégiateurs de l'île. Comme l'a montré notamment Paul Siblot, les positionnements idéologiques marquent les dénominations des noms propres, qu'on parle de Kiev ou de Kyiv, de Londonderry ou de Derry, de San Sebastian ou de Donostia. Toutes les dimensions sociales trouvent des marqueurs à travers les langues : c'est le cas avec le genre (dont les variantes sont difficiles à situer pour le français, mais semblent trouver une expression en japonais, dans les variances prosodiques et d'honorifiques), l'âge (souvent les expressions de haut degré comme grave, différents termes "argotiques " régulièrement renouvelés du genre taffer), de la filiation nationale (lycée, athénée, secondaire;), ethnique (les Gaulois), religieuse (les goys), régionale (les horzains), ou professionnelle (Si vous en êtes d'accord, je suggère que nous procédions à l'examen des dispositifs proposés).

Ces variables lexicales, comme les autres variables, montrent que la variation est un fait de réseaux. C'est un des multiples groupes auxquels appartiennent les locuteurs qu'indexe la variation.

\section{Théorie de Milroy}

7 Une approche articulée de cette question s'appuyant sur un solide fondement empirique est donnée par la théorie sociolinguistique des réseaux sociaux, de Lesley Milroy (1987 notamment). Selon cette théorie, ce qui détermine les variantes utilisées par un locuteur, ce sont les réseaux dans lesquels il s'insère et la force des relations qu'il y établit. Ce cadre permet de prédire comment évoluent et se diffusent les variables à travers une communauté. L'étude de Lesley et James Milroy réalisée dans les années 1970 repose sur une méthode d'observation participante. Ils cherchent à corréler la prononciation de 6 traits phonologiques de l'anglais irlandais selon les communautés catholiques et protestantes et le type de réseaux auxquels les locuteurs appartiennent. Ils arrivent à distinguer les réseaux denses 圈 constitués de relations dites multiplexes et stables entre individus typiquement à mobilité géographique et sociale réduite qui tendent à manifester un comportement linguistique conservateur et à ne pas admettre facilement les innovations - et les réseaux clairsemés - constitués de relations uniplexes et variables entre individus de mobilité plus importante qui tendent à créer et diffuser l'innovation. Au-delà des barrières entre les deux groupes, certains traits utilisés par les femmes d'un des quartiers ouvriers catholiques se répandent auprès des locuteurs de quartiers ouvriers protestants parce que ces femmes travaillent dans les magasins des quartiers protestants et y entretiennent avec les locuteurs de ces quartiers de nombreuses relations uniplexes - elles participent à un réseau ouvert.

8 Selon Milroy, des interactions fréquentes entre des individus entretenant un lien faible, typiquement des interactions commerciales, sont donc susceptibles de diffuser des variables linguistiques nouvelles. C'est l'idée que retient Boerm (2008) dans une étude diachronique de l'évolution de la négation du français. En effet, la négation à double

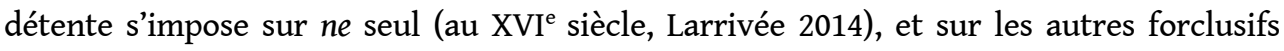
comme mie ou point. L'idée reçue est que l'origine du changement linguistique serait régionale et s'accomplirait à Paris. Ce que Boerm conteste. Il fait valoir que les chartes parisiennes au XIII siècle sont en retard pour différents phénomènes d'évolution historique comme l'ordre des mots SVO et que les forclusifs y sont mieux représentés par 
mie que par pas. Au contraire, pas est bien représenté dans certaines langues frontalières, notamment le Catalan. L'hypothèse que propose Boerm est que le pas du catalan serait à l'origine de l'adoption de ce forclusif en français. Mais par quels réseaux le marqueur peut-il passer de Catalogne au Nord de la France? Comme pour les Milroy, ce serait par les relations ouvertes uniplexes du commerce. Le vecteur de ce changement serait les marchands juifs expulsé du Sud de la France dans la dernière partie du XIII ${ }^{e}$ siècle. Les Juifs formeraient en effet une partie importante des marchands; or dans le Comtat Venaissin, au XIV ${ }^{e}$ siècle, 87 des 94 marchands sont juifs. L'examen de la négation dans des textes réputés appartenir au judéo-français donne des résultats allant dans le sens d'une variété où domine pas chez des locuteurs appartenant à des réseaux ouverts, qui seraient donc responsables de la propagation de la variable. Le tableau suivant résume les données assemblées par Boerm :

\begin{tabular}{|c|c|c|c|c|c|}
\hline Texte & Date & Région & Type & $\begin{array}{l}\text { Ne seul / pas / autres } \\
\text { forclusifs }\end{array}$ & Notes \\
\hline $\begin{array}{l}\text { Une lettre de } \\
\text { Deux Élégies du } \\
\text { Vatican }\end{array}$ & 1288 & ? & Essai? & $7 / 3 / 0$ & \\
\hline $\begin{array}{l}\text { Chansons } \\
\text { Hébraïco- } \\
\text { Provençale }\end{array}$ & $\begin{array}{ll}\text { Du } & 13^{\mathrm{e}} \\
\text { au } & 15^{\mathrm{e}} \\
\text { siècle }\end{array}$ & Provence & Chansons & $2 / 3 / 1 \mathrm{ges}$ & \\
\hline La Reine Esther & 1330 & Provence & $\begin{array}{ll}\text { Pièce } & \text { de } \\
\text { théâtre } & \end{array}$ & $\begin{array}{l}26 / 76 / 17 \text { gis, } 1 \text { mie, } 1 \\
\text { point }\end{array}$ & $\begin{array}{l}44 \text { des } 76 \text { pas sans } \\
\text { négation } \\
\text { préverbale }\end{array}$ \\
\hline $\begin{array}{ll}\text { Cants de } & \text { noces } \\
\text { dels } & \text { jueus } \\
\text { catalans } & \end{array}$ & \begin{tabular}{|l} 
Entre \\
1340 et \\
1420
\end{tabular} & Catalogne & \begin{tabular}{|l} 
Jewish \\
wedding \\
songs from \\
Cataluña
\end{tabular} & Fréquent / 9 / ? & \\
\hline $\begin{array}{l}\text { Une lettre du } \\
\text { rabbin } \\
\text { Brodot du } \\
\text { Rabbinat de } \\
\text { Metz }\end{array}$ & 1432 & Metz & Lettre & $? / 3 / 0$ & $\begin{array}{l}\text { Dans une région } \\
\text { où mie domine }\end{array}$ \\
\hline $\begin{array}{lr}\text { Lettres } & \text { du } \\
\text { rabbin } & \text { Rabbi } \\
\text { Schwab dans } \\
\text { Jewish } & \text { Letter } \\
\text { Exchanges }\end{array}$ & 1567 & Metz & Lettres & $\begin{array}{l}\text { Exclusivement pas sauf } \\
1 \text { point }\end{array}$ & \\
\hline Lis Obros & $\begin{array}{l}\text { Autour } \\
\text { de } 1600\end{array}$ & Provence & $\begin{array}{l}\text { Hymnes } \\
\text { religieux }\end{array}$ & $0 / 10 / 3$ ges & 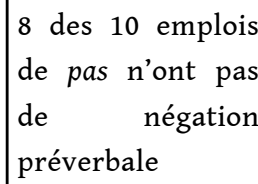 \\
\hline
\end{tabular}




\begin{tabular}{|l|l|l|l|l|l|}
\hline Piyouts & $\begin{array}{l}\text { Autour } \\
\text { de } 1700\end{array}$ & Provence & $\begin{array}{l}\text { Hymnes } \\
\text { religieux }\end{array}$ & $\begin{array}{l}\text { Seulement pas comme } \\
\text { forclusif }\end{array}$ & \\
\hline
\end{tabular}

Et Boerm de conclure

The fact that Jews were primarily merchants in the Middle Ages placed them squarely at the epicenter of linguistic change. The scenario I lay out in this dissertation is not the first to discover the role of commerce in language change. It has been shown that merchants have played a significant role in the linguistic developments of other countries, in England for example. In the history of English it was the merchants of London whose particularities of the East Midland dialect eventually became the accepted forms in standard language. For instance, the noun 'mill' was pronounced / $\mathrm{mIl}$ / by merchants, but /məl/ by the lower-class people of London whose speech would evolve into the Cockney dialect (Leith 1983: 38). East Midland characteristics were imported into London from other towns like Leicestershire with the emergent mercantile class in the 13th century. Just as the Jewish merchants of France perpetuated the spread and acceptance of Mediterranean pas, so the merchants of London did the same for East Midland speech which "would have been heard, and possibly used as a kind of lingua franca among a mobile group". (Leith 1983: 39). (Boerm 2008 : 258)

Le travail soulève un certain nombre de questions. Le nombre de textes, de types et de régions hétérogènes, est faible, et faible le nombre d'occurrences. Les sources tardives ne sont pas concluantes : elles pourraient très bien démontrer l'adoption d'une variante déjà adoptée par la langue générale. A époque précoce, des langues régionales comme le français de Normandie utilisent presque exclusivement le pas, et ce avant l'expulsion des Juifs de la fin du XIII ${ }^{\mathrm{e}}$ siècle. Enfin, alors que les relations entre marchands catalans et l'Italie du Nord étaient apparemment significative, les dialectes italiens concernés n'adoptent pas le forclusif pas.

11 Boerm entend toutefois à juste titre montrer que la variation et le changement se créent et se diffusent à travers l'interaction communautaire. L'interaction se fait dans le cadre des réseaux auxquels participe le locuteur. Ces réseaux en viennent à marquer le langage utilisé dans leur cadre. L'étude a l'avantage de montrer comment l'analyse des réseaux peut tirer parti d'une méthodologie de corpus. C'est ce que font aussi les contributions au présent numéro de Corela.

\section{Présentation des contributions}

L'objectif de ce dossier sur les « Réseaux communautaires et variation dans le langage en moyen français et français préclassique » est d'aborder la variation dans le langage comme signe d'appartenance à un groupe d'individus. L'idée est que les choix de langage signalent l'adhésion d'un locuteur à un réseau communautaire. L'approche est linguistique mais veut s'appliquer à des corpus de tout type. Dans la mesure où elles permettent de suivre la constitution et la progression de réseaux communautaires pour une langue bien documentée comme le français, les ressources anciennes offrent en l'occurrence une opportunité de faire l'archéologie des pratiques d'écriture en réseau. Il s'agit ainsi de voir dans la tradition textuelle, littéraire ou non, de cette large période (c. 1400-1650) le processus de constitution d'une écriture communautaire. 
13 Pascale Mounier analyse Les enjeux de l'emploi communautaire de motifs séquentiels en analyse $d u$ discours. L'observation de départ est que certains récits du seizième siècle portent à l'attention des expressions récurrentes et qui leur semblent propres. On note par exemple l'expression «impareil mariage " pour stigmatiser les unions entre une jeune épouse et un mari âgé. Ces expressions sont-elles l'indice d'un échange réel entre des auteurs parfois inconnus? Que les œuvres étudiées soient publiées à Lyon pourrait le laisser croire. Ce dont on peut être certain, c'est que le roman et les nouvelles marqués par ces expressions définissent une même éthique amoureuse : celle selon laquelle l'amour est affaire de jeunes gens et de jeunes personnes, qui doivent suivre la pente de leur sentiment. Dès lors, c'est une idéologie commune, une connivence idéologique, que marquent les expressions partagées par ces textes, qu'il y ait contact réel ou non entre leurs auteurs.

14 Isabelle Bretthauer étudie pour sa part le système graphique et communauté des "professionnels de l'écrit »: analyse linguistique du manuel de Guillaume Flambart (c. 1470). Ce travail s'inscrit dans son programme de recherche sur les modalités d'apprentissage de l'écrit professionnel par les clercs. Il se penche sur le manuel qu'un noble normand a produit à son propre usage dans sa pratique d'officier des forêts. Venu à la profession sur le tard, Flambart met en œuvre des pratiques l'inscrivant dans la communauté des spécialistes de l'écrit légal, tout en l'en démarquant (voir les nasales en $m$ ). Cette pratique mi-idiosyncrasique et mi-systématique de l'écrit par un officier de l'administration constitue un témoignage important du mouvement général de normalisation de la rédaction d'actes juridiques.

Le travail de Caroline Laske porte sur l'évolution du français en Angleterre comme marque d'adhésion au réseau communautaire du common law. Amené par Guillaume le Conquérant, le law french est une langue relativement répandue dans la société, à des niveaux de connaissance diverse selon, suppose-t-on, les classes sociales, jusqu'à la fin du $\mathrm{XIV}^{\mathrm{e}}$ siècle. Il est notamment utilisé comme langue de l'institution judiciaire, domaine dans lequel elle subsiste jusqu'au XVIII ${ }^{e}$ siècle, bien au-delà de l'anglicisation des autres institutions politiques et administratives. L'étude montre comment une langue travaillée pour des fins professionnelles devient le signe de distinction d'un groupe social. Le law french, qui a marqué l'anglais est ainsi une langue de spécialité qui singularise la société des juristes britanniques et qu'ils n'ont abandonnée qu'à regret, comme le montrent les témoignages réunis.

16 Enfin, Miriam Speyer s'intéresse à L'ombre de Caliste. Le sonnet galant écrit à partir de Malherbe. Elle analyse des récurrences de termes à la rime dans la poésie galante de François de Malherbe et d'imitateurs, conscients ou non, de celle-ci. L'identification de certaines caractéristiques du travail malherbien, notamment la postposition du forclusif de négation pas à des infinitifs alors que cette façon de dire est en train de passer, permet d'estimer le degré d'adhésion de ses contemporains au classicisme du modèle. Il s'agit donc d'envisager les réseaux non pas comme des pratiques absolues, mais comme des engagements relatifs selon le degré d'accord à une démarche collective.

17 Ainsi, les contributions concernent des processus touchant différents aspects de la dimension communautaire de l'emploi du langage. C'est l'insertion dans une communauté idéologique et esthétique que donnent à voir les traits linguistiques différenciés mais récurrents dans les études de P. Mounier et de M. Speyer. En-deçà des choix stylistiques que permettent ces productions littéraires, un effort individuel d'acquisition de la langue légale est en jeu dans l'analyse de I. Bretthauer. Enfin, la langue 
de spécialité comme marqueur d'un groupe professionnel fait l'objet de l'étude de C. Laske. Ces quatre analyses de cas entendent encourager, c'est le souhait des coordinateurs de ce dossier, la recherche de typologies englobantes pour la compréhension du rôle des rapports communautaires dans l'évolution de la langue et l'écriture de textes.

\section{BIBLIOGRAPHIE}

Behnstedt, Peter (1978), Viens-tu ? Est-ce que tu viens? Tu viens ? Formen und strukturen des direkten Fragesatzes im Französischen, Tübingen, Narr.

Boerm, Michael Lloyd (2008), Pourquoi 'Pas': The Socio-Historical Linguistics Behind the Grammaticalization of the French Negative Marker, Thèse de doctorat, University of Texas at Austin.

Iglésias, Olivier et Larrivée, Pierre (2014), « Une approche idiolectale de la chute de ne en français contemporain », in Congrès Mondial de Linguistique Française 2014, dir. Franck Neveu, Peter Blumenthal, Linda Hriba, Annette Gerstenberg, Judith Meinschaefer et Sophie Prévost, mise en ligne SHS Web of Conferences. 2397-2411.

Labov, William (1987), « How I got into linguistics, and what I got out of it ». mise en ligne http:// www.ling.upenn.edu/ wlabov/HowIgot.html.

Larrivée, Pierre (2014), « Reanalysis of negatives as polarity markers? The last 400 years of decline of the French preverbal negative clitic », Lingua, $n^{\circ} 147$, p. 40-49.

Lefeuvre, Florence et Rossi-Gensane, Nathalie (2015), Interrogation. Projet FRACOV (FRAnçais COntemporain Vernaculaire), mise en ligne http://www.univ-paris3.fr/index-des-

fiches-227311.kjsp.

Levinson, Stephen C. (2006), « On the human "interaction engine"”, in Roots of human sociality: Culture, cognition and interaction, dir. N. J. Enfield et S. C. Levinson,. Oxford, Berg.

Milroy, Lesley (1987), Language and social networks, Oxford, Basil Blackwell.

Saussure, Ferdinand de (1972), Cours de Linguistique générale, Paris, Payot.

\section{RÉSUMÉS}

Cette présentation rappelle certains des paramètres centraux de l'analyse de la variation linguistique. Si la variation apparaît contredire l'idée d'un système linguistique homogène, elle n'en reste pas moins un phénomène social: elle ne se confond pas avec les productions idiolectales, qui elles-mêmes, si elles sont compréhensibles, exploitent des potentialités du système linguistique. Les différents groupes auxquels peut être affilié un locuteur sont concernés par la variation grammaticale et lexicale, et les variables peuvent servir à indexer l'appartenance au groupe. C'est ce dont rend compte la sociolinguistique des réseaux de Lesley Milroy, dont une application récente à la diachronie du français est discutée. L'usage de corpus pour évaluer les 
réseaux sociaux qui sous-tendent la variation historique est le thème de ce numéro dont les contributions sont présentées.

This opening chapter reviews central dimensions in the analysis of linguistic variation. The existence of linguistic variation runs counter to the structuralist idea of a homogeneous linguistic system. It illustrates the social dimension of language, as the contrast with idiolectal practices shows. Linguistic variation correlates with the different groups to which each speaker is affiliated to, and affiliation claims can be performed by use of variables. The perspectives offered by Network Sociolinguistics developed by Lesley Milroy are considered in view of a recent study of the diachrony of French. The use of corpora to assess social networks that underlie historical variation is the theme explored in the contributions to this issue that are each introduced.

INDEX

Mots-clés : variation, sociolinguistique, sociolinguistique des réseaux, diffusion des changements diachroniques

Keywords : Variation, sociolinguistics, network sociolinguistics, diffusion of diachronic change

\section{AUTEUR}

\section{PIERRE LARRIVÉE}

Université de Caen Normandie / EA 4255 CRISCO 